\title{
The place of psychotherapy in modern psychiatric practice ${ }^{\dagger}$
}

\section{Chess Denman}

\begin{abstract}
SUMMARY
This article makes a case for training and developing psychotherapeutically competent consultant general psychiatrists. It describes what such psychiatrists should know and suggests that they should be able to prescribe psychotherapy rationally and assess its outcome. They should be able to deliver some psychological treatments themselves but, more importantly, they should be competent to apply psychotherapeutic knowledge and emotional literacy skills to their routine clinical practice. Possible CPD experiences are suggested to increase the competencies of existing consultant general psychiatrists.
\end{abstract}

\section{DECLARATION OF INTEREST}

None.

Many psychiatrists value the contribution of psychological thinking and treatments to their discipline but few are expert in this area and many have had much less training in this than in psychopharmacology. A survey by the Postgraduate Medical Education and Training Board (2010) shows that modern trainees remain more confident of receiving adequate training in psychopharmacology than in psychiatry.

\section{Neglect of psychology and psychotherapy}

One reason for the neglect of psychology and psychotherapy in psychiatry may be a failure to understand and to argue for the value of medical psychotherapy. Psychological treatments are viewed as something easily left to psychologists and other practitioners. This is a mistake. It embodies an erroneous view of psychological treatment as a non-medical intervention with no side-effects and no interactions with other treatment. The rational prescribing and evaluation as well as the delivery of psychological treatments requires medical input. The mistake also represents a failure to understand that psychotherapy is not just about treating patients in formalised settings. It is also a way of conceptualising, orchestrating and conducting the treatment of all patients, as is an understanding of their social circumstances and biochemistry. A psychologically unsophisticated management plan may neglect critical issues such as adherence to medication or reluctance to attend appointments because of the humiliation of being unwell.

There are failings at a theoretical level too. One of the singular achievements of psychiatry was to develop, and in many cases apply, the complex and subtle account of the pathogenesis and expression of mental disease that is termed the 'biopsychosocial model'. It is ironic, therefore, that among psychiatrists the psyche is the neglected part of this analysis. Psychiatrists expect themselves to be expert psychopharmacologists, and crucial developments in the social organisation of the delivery of care and the process of rehabilitation have been driven forward by a powerful social model that stresses the importance of social forces both in normal development and in the pathogenesis of psychiatric ill health. Yet the 'psycho' element in the biopsychosocial model seems to have dropped out in psychiatry.

To the extent that it is true, why might this be the case? One reason could be the failings of psychotherapists and psychologists to give an account of their discipline that is at once unified, credible and practical. Arcane disputes between rival therapeutic schools (modality wars!) and complex training procedures serve to lock psychotherapeutic understandings into a professionalised isolation. Another difficulty might be that psychiatrists have wanted to maintain close links with medical colleagues and have seen non-physical methods of treatment as only secondary or adjuvant to their concerns.

In this article I shall argue that all psychiatrists should be deeply concerned about both the generalised lack of real involvement in psychotherapy as a branch of psychiatric treatment and the failure to engage with the theoretical approaches that psychotherapy and psychology bring to the study of psychiatric illness. The training and development of psychotherapeutically literate psychiatrists must be brought to the fore or psychiatrists risk becoming at best biosocial practitioners or at worst pill-pushing agents of social control.
Chess Denman is a consultant psychiatrist in psychotherapy in charge of the Complex Cases Unit in Cambridge, UK, which treats patients with personality disorder. She is Chair of the Education Committee of the Faculty of Psychotherapy at the Royal College of Psychiatrists and is responsible for coordinating revisions to the new curriculum of training for psychiatrists as it relates to psychotherapy.

Correspondence DrChess Denman, Complex Cases Service, Springbank Ward, Fulbourn Hospital, Cambridge CB21 5EF, UK. Email: chess.denman@cpft.nhs.uk

'See pp. 240-242, this issue. 


\section{What should a good consultant know about and know how to do?}

\section{Prescribing formal psychotherapy appropriately}

Psychiatrists and service planners have correctly left the treatment of patients requiring specific forms of psychotherapy to non-medical specialists. The only exception to this has been some forms of dynamic psychotherapy, which, rather as a matter of historical accident, have chiefly been practised in the National Health Service by consultant psychotherapists and their staff. However, as overseers of treatment psychiatrists have been left in the role of 'prescribers' of such interventions. As such, a knowledge of the intervention, its indications, contraindications, side-effects and interactions with other treatments must be part of the appropriate knowledge base for consultants.

\section{Potential adverse effects of psychotherapy}

For each of the adverse effects listed in Box 1 a sensitive and psychologically informed case assessment will reveal both systematic and individual factors that may predispose a patient to adverse effects and represent contraindications to therapy. At times, referral for an expert opinion and assessment will, of course, be appropriate. However, there are potential adverse effects from referral for assessment. Many patients arrive without a clear understanding that they are being offered assessment rather than treatment. A refusal to treat can come as a very serious blow.

\section{Case study 1}

Petra was referred from psychiatric out-patients and attended for assessment at the local psychotherapy department, expecting to start therapy at once. The referral letter gave no formal psychiatric history referring to care programme approach (CPA) documentation (which was not enclosed) but mentioned a

BOX 1 Potential adverse effects of psychotherapy

\section{Major}

- Malignant regression and suicide

- Psychotic episodes

- Increased depression and hopelessness

- Rash or irresponsible behaviour

- Increased risk of aggression and assault

Minor

- Cost and inconvenience of weekly sessions (travel, time off work)

- Disphoric emotions and increased self-doubt

- Disrupted interpersonal relationships need to 'work through issues related to early sexual abuse'. Unknown to her referrer, Petra was already receiving art therapy and was also consulting a private counsellor but felt she 'needed more input'. A history of her previous psychotherapeutic experiences revealed that she had in the past had a lengthy treatment that had to be terminated when she began to stalk her therapist. Petra was disappointed when the consultant psychotherapist tried gently to explain why further treatment might not be advisable and was angry that she had been referred when 'nothing was going to be offered'.

Difficulties do not end here. Psychological treatments are not like medications. For one thing there are no drug companies ensuring the quality of the intervention. Referrers need to understand that a course of cognitive-behavioural therapy can mean a highly specialised treatment offered by a professional with a 3-year training in cognitive-behavioural therapy and 10 years' experience in its practice, or an intervention by a staff member who has attended a course in behavioural interventions for two terms of one half-day a week.

Furthermore, there are a bewildering array of psychological treatments. If a specialist psychotherapist with experience of and access to a range of different psychotherapeutic modalities is available and appropriately resourced then they may be able to appropriately select the modality and quality control the intervention. However, such individuals, medical or otherwise, are thin on the ground. It is therefore left to the prescriber to ensure some form of quality control and, often in those parts of the country lucky enough to be able to offer choice, to select the modality (by virtue of the person to whom they refer).

\section{Case study 2}

Philip was referred for cognitive-behavioural therapy to the local psychological treatment service. The letter was brief and referred to anxiety and problems at work. He was taken on by a trainee psychologist with the aim of offering standard anxiety management. Some weeks into treatment it was clear that Philip's problems were long-standing. He was socially phobic, living with his mother despite being 40 , and was chronically anxious and tormented by the fact that he had never had a sexual relationship. His difficulties at work had been precipitated by distress caused by being moved to work under a female manager. In supervision it became clear that the trainee psychologist was out of her depth, not least because Philip soon developed sexual ideas about her.

\section{Applying psychotherapeutic knowledge to routine clinical practice}

Even more destructive to the accurate practice of psychiatry than problems in referral is the commonly held view that psychotherapy is 
something done by specialised individuals in pleasant consulting rooms far away from the practice of general psychiatry. This is far from true. There is ample evidence that a psychological approach is an essential element in all areas of psychiatric practice.

\section{Quality of care and the risk of suicide}

Consider the measures needed to deal with the risks of suicide on or shortly after discharge from acute wards. In-keeping with a realisation that the final moments before a suicide are often impulsive, much work has been done on ward environments. However, poor continuity of care is also associated with increased suicide risk in in-patients (Desai 2005). Why? After all, the modern care programme approach (CPA) should ensure the seamless replaceability of members of the staff team in carrying through a plan of management. Perhaps the CPA can ensure continuity of planning and transmission of critical information but cannot take account of the important, often highly dependent, attachment relationships that patients form while on the ward. Deprived of secure attachment figures patients are less likely to speak their minds; suicidal thoughts and plans may simply not be revealed. Planning and paying attention to the attachments that a patient forms while an in-patient (ensuring, for example, that the patient's named nurse and the senior house officer involved are not both about to go away on holiday the next day) may not sound like psychotherapeutic tasks. However, the theory behind them (attachment theory) can, when sensitively and accurately applied, identify patients who are likely to speak their minds freely and those who are not. Such a perspective is self-evidently vital to the accurate assessment of suicide risk.

Even with the best management in the world, suicides will occur and when they do they will have impacts - often adverse - on the staff involved. Units may well have developed policies for debriefing after such incidents. However, there is also evidence that other patients on a ward where a suicide has occurred become upset and their responses are often ignored (Bowers 2006).

The necessity of managing suicide correctly makes it a helpful headline topic for highlighting the importance of a psychotherapeutic approach to routine clinical care, but there are many other areas where such an approach is vital to accurate management (Box 2).

\section{Understanding and managing burnout and boundary violations}

No professional sets out to act in an unprofessional way and yet unprofessional behaviour does occur.
Psychotherapeutically informed thinking can contribute much to the analysis and prevention of unprofessional behaviour by staff.

\section{Case study 3}

Jane has severe recurrent depression with psychotic features but will never consent to be admitted to hospital again. She stated that during her last admission a patient stole her reading glasses from the locker beside her bed. When she asked a nurse for help finding them she was told sharply that she ought to 'take responsibility for her own possessions'. Jane felt furious, patronised and terribly to blame for the offending patient's subsequent severe self-harm with her broken glasses.

The problem is to reduce the number of experiences such as Jane's. The nurse's reaction to Jane's predicament represented a small act of hostility that may have been unthinking or that may have been an irritable response to agitated depression, which can be very wearing to work with because the patient can often be distressed and asking for help but not reassurable. The nurse's response is also a small example of a boundary violation committed in a moment of burnout.

\section{Insight from a psychotherapeutic perspective}

A crucial insight that a psychotherapeutic approach can bring to this area is to remedy the tendency to see burnout as a condition resulting from the individual emotional vulnerability of a single member of staff who has some kind of emotional crash-landing. A psychotherapeutic approach can help those involved to understand that burnout represents the outcome of powerful emotional forces, not completely under conscious control, to which all staff are subject.

\section{BOX 2 Psychotherapeutically informed practice}

Areas of psychiatric practice in which a psychotherapeutically informed approach is essential include:

- addictions and eating disorders

- personality disorders, somatisation disorders and other complex cases

- adherence to medication

- psychoeducation and relapse prevention

- help for carers

- treatment-resistant disorders

- management of difficult or conflictual relationships with patients

- patients with whom it is hard to communicate or make an alliance 
These forces derive in part from our nature, in part from the extremities of psychiatric illness and in part from the contradictory expectations of our culture (which values individual autonomy but also demands safety for the many from the few conceived of as 'mad'). Were psychiatrists to be more conscious of these forces, they would be less surprised and less repeatedly caught off-guard by the major sexual and aggressive boundary violations that, while infrequent, cannot now be thought of as unusual in medicine.

These large events are not as rare as might be hoped. They represent the tip of an iceberg of smaller repeated minor infractions and unprofessional moments. It is now known that aggressive emotions towards patients (Holmqvist 2006) and difficulty in personal life (Gabbard 1996) are two key factors that predispose to both burnout and boundary violations. Psychotherapeutically competent psychiatrists can monitor themselves in this area and help to maintain oversight of the medical and multidisciplinary team by careful but non-intrusive closeness to staff and sensitive handling of the situations that present.

\section{Case study 4 \\ Garreth was an 83-year-old man with fairly profound dementia. He was found one morning to have a dislocated shoulder. No fall or trauma had been recorded. It transpired that the injury had been sustained when he was roughly dragged up in bed while being cleaned in the night having been faecally incontinent. The staff member concerned, L, had considerable personal difficulties at home and was well known for her judgemental attitude to patients who 'caused difficulties'. Her line manager was aware of L's personal and professional difficulties but felt powerless to act. As a result, Garreth was injured and L was severely disciplined. Perhaps a frank conversation and even the suggestion that $\mathrm{L}$ might not be suited to this kind of work would have helped.}

\section{Delivering education and training}

Consultants are expected to teach and mentor their junior staff. The structure of weekly educational supervision and clinical apprenticeship provides an arena in which consultant psychiatrists can have an enormous impact on their trainees' knowledge, clinical skills and attitude. Yet psychotherapy education has been restricted to a little cordoned-off area of learning where doctors repair to the psychotherapy department for training.

Educational supervisors have, perhaps too often, accepted that they are not skilled enough to take on some of this training. In doing so they have contributed to the fundamental lesson that all trainees who have been lucky enough to receive psychotherapy teaching have been given: that psychotherapy is something done 'over there' by 'them' - an odd bunch with eccentric ways and little knowledge of or contact with the real world of acute psychiatric practice. Gabbard (2005) lists this as the prime error that educators can commit.

Psychotherapeutically competent consultant psychiatrists and educational supervisors would be able to teach at a minimum the application of basic psychotherapeutic principles to routine clinical practice. They would be aware of the necessary psychotherapy competencies that their junior doctors need during their training. Competent educational supervisors should be able and confident enough to comment on the human and emotional skills and qualities of the trainee as well as their clinical skills and theoretical knowledge. Knowing the local resources and working in conjunction with the local psychotherapy tutor and scheme organiser, the educational supervisor should be able to assist the junior doctor in obtaining the training experiences that are needed to develop the competencies.

\section{Case study 5 \\ F travelled regularly to a distant site to offer 'psychotherapy supervision' to the juniors. Each week a different subgroup attended and were keen, motivated, intelligent and questioning. Initially, F ran a case-based discussion group but it was clear that whenever the diagnosis was of a major psychiatric disorder such as bipolar disorder or schizophrenia, the group had the greatest difficulty in thinking about psychosocial influences on the case in terms other than adherence to medication. After some time, many of them wanted to treat patients under supervision. F agreed but no patients could be found to treat. The psychology department felt the trainees to be too junior and had their own trainees to provide with patients. The community mental health teams and the consultants working in out-patients never seemed to have any 'suitable' patients. Conversation between $\mathrm{F}$ and the consultants revealed that the stereotyped notion of a suitable patient was of an employed, mildly depressed, middle-aged individual with interpersonal difficulties of the sort no longer seen in secondary care.}

\section{Being involved in planning and management}

The organisation of services at F's hospital brings to the fore the issue of planning and management.

\section{Case study 6}

Staff retirement left $\mathrm{P}$ on his own in a large trust as the only psychotherapy consultant. He tried to gather support so that consultant sessions should not be lost, but the trust had many other priorities and was short of money. A multidisciplinary review of psychological treatment services was set up to investigate the allocation of resources. The medical director was supportive but busy and strongly 
encouraged $\mathrm{P}$ to advocate, saying 'You're our man on that committee'. When $P$ asked in a consultants' meeting for an adult consultant to join him in the review group there were no volunteers, but $\mathrm{P}$ was supported with the telling phrase "We're right behind you'. By contrast, in R's trust the clinical director herself convened and chaired a working party aimed at improving psychological treatment services and removing gaps in provision.

Of course, being 'right behind' a local consultant psychotherapist is not an option for many acute psychiatrists because there is no psychotherapist to support. For this reason if no other, intelligent service planning in this area has to be part of the role of acute psychiatrists. In any case, psychotherapeutically competent general psychiatrists who took seriously the provision of formal psychotherapy and the promotion of a psychotherapeutic approach to clinical management simply would be unable to restrain themselves from becoming involved in the management and quality control of these services. Debates in this area would be as heated and as swiftly engaged as those that ensue when restrictions are suggested for prescribing some pharmacological agents after national or local formulary restrictions are in prospect. A competent psychiatrist would want to make sure that they were aware of the current state of provision within the local organisation. They would have some knowledge of alternate models of service provision and awareness of key documents such as Role of the Consultant Psychiatrist in Psychotherapy (Royal College of Psychiatrists 2006). This knowledge would equip them to advocate rationally for psychotherapy provision that suited their trust.

\section{From competencies to curriculum}

The wish list of competencies that I have defined so far sets a curriculum for the acquisition of those competencies in the training of future consultant psychiatrists and their promotion and maintenance in the current consultant workforce. The details of this curriculum are beyond the scope of this article but certain broad features are apparent.

\section{Academic knowledge}

As it is for pharmacology, so it is with therapy. Consultants should be able to talk knowledgeably about the different classes of therapy available and be aware of their mechanism of action and likely outcomes, successful and otherwise. Consultants should have a good knowledge of the main psychological theories that account for a range of psychological conditions and reactions. They should know what is needed to refer intelligently, tell the patient what treatment will involve, follow up the outcomes of treatment and have a knowledge of second-line interventions. Consultants should know enough about the theory of group interactions and human relationships to be able to analyse the likely outcomes of situations that arise in multidisciplinary teams and other areas in the workplace. They should be aware of theory-based management strategies for dealing with staff conflict. All of this understanding should be based on a sound and up-to-date knowledge of the expanding evidence base in psychological treatments.

\section{Clinical skills}

Consultants need to have the skill of assessing and referring patients for psychological treatments, which includes being able to ensure that the patient understands the treatment being proposed and has a sanguine perspective on its advantages and disadvantages. Being able to deliver simple but effective psychotherapeutic interventions during routine clinical practice or in a small case-load of formal therapies is another easily acquired skill with high utility - psychotherapy really isn’t rocket science.

Much more complex and taxing are the psychotherapeutic skills needed to practise good acute psychiatry. At the core of these is the capacity to sense, tolerate, reflect on and act appropriately in relation to strong feelings in the self and others. This is known as emotional literacy.

\section{Emotional literacy}

The idea that anyone else, and consultant psychotherapists in particular, has specific emotional literacy skills that consultant psychiatrists should attempt to emulate is a difficult one for a couple of reasons. First, it is quite emotionally difficult to accept that other people might be more sensitive, or kind or compassionate than oneself and these are the qualities that are generally associated with emotional literacy. Second (and this comment is always rather sotto voce), many practitioners could name a psychotherapist or two whose emotional literacy seems a little on the low side. This may account for the finding that whereas medical students respond relatively well to the teaching of communication skills (Smith 2007), experienced physicians may not do so (Hulsman 1999). However, this view of emotional literacy assimilates the competency to the 'soft skills' of politeness and consumer management taught by businesses. The difficulties that psychiatrists often have to wrestle with tend to involve anger, distrust and defeat. Maintaining long-term relationships with ambivalent or conflicted patients and with staff 
MCO answers

$\begin{array}{lllll}1 d & 2 d & 3 a & 4 b & 5 c\end{array}$
BOX 3 More advanced emotional literacy skills

- Treating patients you hate without over- or undercompensating

- Explaining to patients that the optimum treatment for them is not available because of resource constraints without burdening the patient unduly

- Recognising emotional exhaustion and managing it

- Writing a fair reference on a poor trainee

- Dealing with a staff member/colleague who possibly has a drink problem

- Maintaining mental closeness with a patient who is seductive or asks personal and intrusive questions without becoming overinvolved or defensive

who are perhaps unsuited to their role are more advanced emotional literacy skills (Box 3).

\section{Case study 7}

One patient had exhausted the resources of a specialist affective disorder unit and a local psychotherapy service. In an abrupt fashion after a dramatic and near-fatal suicide attempt she discontinued treatment and refused all support and input from anyone other than her consultant psychiatrist. The psychiatrist maintained contact in out-patients and over a period of time was able to offer some low-key cognitive-behavioural therapy framed as strategies for coping as part of regular out-patient appointments. The patient settled.

\section{Maintaining and improving clinical skills}

Just as academic knowledge in psychotherapy progresses, so there are important advances in technique. In the past 30 years a range of important, exciting but theoretically and technically challenging treatments have been developed, often with acute psychiatric settings in mind. Consultant psychiatrists should update their clinical skills in assessment and treatment to take account of this and of future expansions (Box 4).

BOX 4 New and promising treatments

- CAT - Cognitive analytic therapy (Ryle 2002)

- DBT - Dialectical behaviour therapy (Linehan 1993)

- SFT - Solution-focused therapy (O'Connell 2005)

- SFT (the other one) - Schema-focused therapy (Youn 2003)

- MBT - Mentalisation-based therapy (Bateman 2006)

- TFT - Transference-focused therapy (Yeomans 2002)

- EMDR - Eye movement desensitization and reprocessing (Shapiro 2001)
The evidence in other areas shows that emotional literacy skills are improved by training but then decline with time. This is a key finding because it is counterintuitive. One would expect such skills to be rewarding and therefore to be employed more frequently and therefore improve because of a practice effect. However, the advanced skills can be aversive to practice and require patience, time and freedom from anxiety.

Refresher training in emotional literacy can be obtained in a large number of ways, not all of which are thought of as 'formal training'. Supervision, mentoring, case discussion groups, collegial chats, reading a good novel and personal therapy can all be useful adjuncts to more formal approaches. The key is to maintain awareness that however good one thinks one is, one's skills are likely to decline with time unless appropriately maintained and it is important to regularly audit the adequacy of input in this area.

\section{Conclusions}

Normally, any discussion of the value of psychotherapy or the need for more psychotherapy training receives warm support followed by words of caution about the difficulty of implementation and shortage of resources. Ultimately, it is a question of priorities. When the Royal College of Psychiatrists took the adequate delivery of safe electroconvulsive therapy (ECT) seriously it was able to take action that substantially raised the standard of training and delivery in this area (Royal College of Psychiatrists 2005). Inaccurate management of patients leads to mortality and morbidity. Although drug errors do occur, most deaths, misdiagnoses and inflictions of iatrogenic mental suffering involve psychotherapeutic and psychological failings. Psychiatrists need to take this thought seriously and raise their personal game through continuing professional development and their collective game by educating future consultants adequately.

\section{References}

Bateman A, Fonagy P (2006) Mentalization-Based Treatment for Borderline Personality Disorder: A Practical Guide. Oxford University Press.

Bowers L, Simpson A, Eyres S, et al (2006) Serious untoward incidents and their aftermath in acute inpatient psychiatry. The Tompkins Acute Ward Study. International Journal of Mental Health Nursing 15: 226-34.

Desai RA, Dausey DJ, Rosenheck RA (2005) Mental health service delivery and suicide risk. The role of individual patient and facility factors. American Journal of Psychiatry 162: 311-8.

Gabbard GO (1996) Love and Hate in the Analytic Setting. Jason Aronson. Gabbard GO (2005) How not to teach psychotherapy. Academic Psychiatry 29: 332-8.

Holmqvist R, Jeanneau M (2006) Burnout and psychiatric staff's feeling towards patients. Psychiatry Research 145: 207-13. 
Hulsman RL, Ros WJG, Winnubst JAM, et al (1999) Teaching clinically experienced physicians communication skills. A review of evaluation studies. Medical Education 33: 655-68.

Linehan M (1993) Cognitive Behavioural Treatment of Borderline Personality Disorder. Guilford Press.

O'Connell B (2005) Solution-Focused Therapy. Sage.

Postgraduate Medical Education Training Board (2010) Trainee Survey 2010: Foundation, Core and Specialty trainee doctors by country compared to UK-wide score. General Medical Council (http://reports. pmetb.org.uk/groupcluster.aspx?agg=AGG39\%7c2010).

Royal College of Psychiatrists (2005) ECTAS: ECT Accreditation Service. RCPsych (http://www.rcpsych.ac.uk/crtu/centreforqualityimprovement/ electroconvulsivetherapy.aspx).
Royal College of Psychiatrists (2006) Role of the Consultant Psychiatrist in Psychotherapy (Council Report CR139). Royal College of Psychiatrists. Ryle A, Kerr IB (2002) Introducing Cognitive Analytic Therapy. Principles and Practice. Wiley.

Shapiro F (2001) Eye Movement Desensitisation and Reprocessing: Basic Principles, Protocols, and Procedures. Guilford Press.

Smith S, Hanson JL, Tewksbury LR, et al (2007) Teaching patient communication skills to medical students. A review of randomized controlled trials. Evaluation and the Health Professions 30: 3-21.

Yeomans FE, Clarkin JF, Kernberg OF (2002) A Primer of TransferenceFocused Psychotherapy for the Borderline Patient. Aronson.

Young J, Klosko JS, Weishaar ME (2003) Schema Therapy: A Practitioner's Guide. Guilford Press.

\section{MCQs}

Select the single best option for each question stem

1 Which of the following is not a genuine new and promising psychotherapy?

a CAT - cognitive analytic therapy

b TFT - transference-focused therapy

c SFT-solution-focused therapy

d IBT - insight-based therapy

e MBT - mentalisation-based therapy.

2 Which of the following are not advanced emotional literacy skills?

a managing malignant regression

b treating patients you hate without over- or under-compensating

c dealing with a staff member/colleague who possibly has a drink problem

d interviewing patients and taking a full psychiatric history e maintaining mental closeness with a patient who is seductive or asks personal and intrusive questions without becoming overinvolved or defensive.

3 Which of the following is a possible major adverse side-effect of psychotherapy?

a malignant regression and suicide

b increased distress after sessions

c persistent psychosis

$\mathrm{d}$ reduced insight

e reduced adherence to medication.

4 Which of the following possible emotional features of acute in-patient care might be associated with increased suicide risk?

a strange and novel environment

b lack of secure attachment figures

c insecure physical environment

d detention under the Mental Health Act

e frequent visits by relatives.
5 Which of the following aspects of psychiatric care is not particularly associated with a need for a psychotherapeutically informed approach?

a personality disorders, somatisation disorders and other complex cases

b psychoeducation and relapse prevention c detaining patients under the Mental Health Act

d management of difficult or conflictual relationships with patients

e patients with whom it is hard to communicate or make an alliance. 